\title{
Effects of combined hardening and free-play nonlinearities on the response of a typical aeroelastic section
}

\author{
Daniel A. Pereira ${ }^{a, 1}$, Rui M.G. Vasconcellos ${ }^{\text {b,2 }}$, Muhammad R. Hajj ${ }^{\text {c,3 }}$, \\ Flávio D. Marques ${ }^{\mathrm{a}, *, 4}$
}

a Engineering School of São Carlos, University of São Paulo, São Carlos, SP, Brazil

b São Paulo State University (UNESP), São João da Boa Vista, SP, Brazil

${ }^{\mathrm{c}}$ Virginia Tech, Blacksburg, VA, USA

\section{A R T I C L E I N F O}

\section{Article history:}

Received 2 April 2015

Received in revised form 18 December 2015

Accepted 19 December 2015

Available online 29 December 2015

\section{Keywords:}

Aeroelasticity

Hopf bifurcation

Flutter

Hardening nonlinearity

Free-play nonlinearity

Higher-order spectra

\begin{abstract}
A B S T R A C T
This paper presents an investigation about the dynamic response of a three-degree of freedom airfoil with hardening nonlinearity in the pitching stiffness and free-play nonlinearity in the control surface stiffness using bifurcation and HOS analysis. An experimental apparatus was conceived to test an airfoil aeroelastic responses when nonlinearities of varying intensities are present. A numerical model is also used to simulate at the same conditions of the experimental tests. It is based on the classical theory for the linear unsteady aerodynamics with corrections for arbitrary motions coupled to a three-degree of freedom typical aeroelastic section, where the hardening effect is modeled by means of rational polynomial function, while the free-play is represented by hyperbolic functions combination. Aeroelastic responses are analyzed from numerical and experimental results. Hopf bifurcations are identified and diagrams of amplitudes versus airspeeds are used to investigate the conditions in which the system is supercritical or subcritical. Higher-order spectra analysis is also used to check on frequency couplings, thereby allowing to identify quadratic- and cubic-like nonlinear behavior. The study of the phenomena associated with the hardening, free-play and their intensity variation effects may be useful in the mitigation of undesired responses of aeroelastic systems.
\end{abstract}

(c) 2016 Elsevier Masson SAS. All rights reserved.

\section{Introduction}

The aeroelasticity is a multidisciplinary field of engineering science that deals with the mutual interaction between structural dynamics and unsteady aerodynamic loading [1]. Aeroelastic systems may behave nonlinearly, therefore exhibiting phenomena such as bifurcations, limit cycle oscillations (LCO), and chaos [2,3]. The source of the nonlinearities can be in structural dynamics and/or the unsteady aerodynamic loading and may be difficult to predict. Nonlinear unsteady aerodynamic loading can be the result of sep-

\footnotetext{
* Correspondence to: Escola de Engenharia de São Carlos, Universidade de São Paulo, Av. Trabalhador Sancarlense, 400, CEP 13566-590, São Carlos, SP, Brazil. Tel.: +55163373 9370; fax: +55 1633739590 .

E-mail addresses: daniel.almeida.pereira@usp.br (D.A. Pereira), rui.vasconcellos@sjbv.unesp.br (R.M.G. Vasconcellos), mhajj@vt.edu (M.R. Hajj), fmarques@sc.usp.br (F.D. Marques).

URL: http://www.eesc.usp.br/fmarques/ (F.D. Marques).

1 Graduate student.

2 Assistance Professor

3 Professor.

4 Associate Professor.
}

arated flows (viscous effects) or shock excursion (compressibility effects). Structural nonlinearities can arise from geometrical of material related effect, and classified as concentrated or distributed. Concentrated structural nonlinear effects can be incorporated into numerical models through the elastic restoring forces or moments representations, being the most common form of nonlinear inclusion to aeroelastic models. Typical concentrated structural nonlinear representations can be given by polynomial fitting functions, nonlinear damping effects, free-play, and hysteresis.

The literature in this field is quite vast, demonstrating that nonlinear aeroelastic problems in the aviation are of increasing importance in aircraft design. For example, the limit cycle oscillations have caused persistent aeroelastic problems in aircraft, such as the F-16, where the existence of hardening nonlinearity in wings' pitching moment stiffness was observed [4,5]. O'Neil and Strganac [6] have developed an experimental test that provides direct measurements from the typical aeroelastic section with cubic nonlinearity in the pitch and plunge motion. They examined the sensitivity of the response to system parameters and provided important conclusions on the effect of smooth nonlinear effect in aeroelastic response of airfoils. Recently, Vasconcellos et al. [7] 

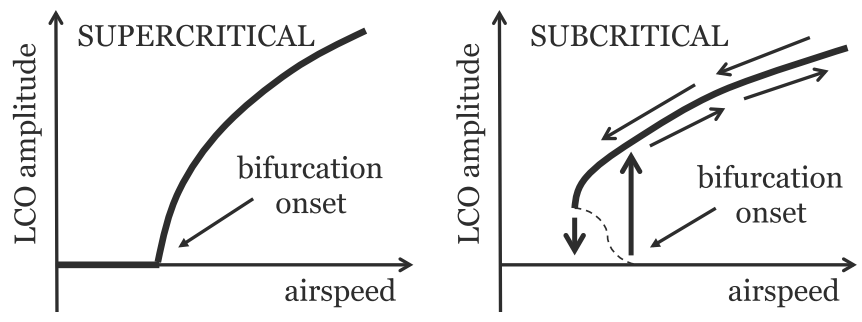

Fig. 1. Hopf bifurcation behavior.

have shown that the hyperbolic tangent function combination approach for modeling discontinuous nonlinearities is appropriate for detecting different nonlinear behaviors, including the experimentally observed LCO, chaos and transitions.

It is known that the system behavior is directly related to the nonlinearities involved, for example, system under free-play shows subcritical behavior [8-11]. The Hopf bifurcation appears when a stable system becomes unstable at certain parameter variation and the attractor becomes a LCO. There are two types of Hopf bifurcation: the supercritical Hopf bifurcation, where stable LCO appears after an unstable critical location, and subcritical Hopf bifurcation, where an unstable LCO is created among a stable critical location. Fig. 1 illustrates the possible behavior of the Hopf bifurcations. Supercritical bifurcations are sometimes called safe because the amplitude of the limit cycles grows up gradually as the parameter is increased after the bifurcation point [3]. In contrast, the subcritical bifurcations are called dangerous because in a subcritical system it is also possible that large-amplitude limit cycles suddenly appear as the parameter is varied. Typically subcritical behavior causes bistable behavior, i.e, there are different solutions when the flow velocity is increased and decreased near the bifurcation point.

Bifurcation analysis can be used to determine quantitative and qualitative changes in the system features, such as the number and type of solutions, under the variation of one or more parameters [12]. An example of this approach for a nonlinear aeroelastic system is given in Ref. [10] that analyzed changes in typical aeroelastic section behavior characterized by a cubic structural nonlinearity. It has been shown that the stability of the system is determined by the influence of the different nonlinear couplings.

In this paper, an investigation on the combined influence of hardening and free-play nonlinearities on the bifurcation response of a typical nonlinear section is presented. Numerical and experimental results are presented, thereby allowing comparisons and conclusions of the nonlinear features. Hardening nonlinearities with varying intensities in airfoil pitching motion and free-play (different gap values) in the control surface hinge were considered. Numerical model includes classical linear unsteady aerodynamics, while the equations of motion incorporates nonlinearities in the respective stiffness values. Traditional time integration method is used in aeroelastic simulations. The experimental apparatus was designed to allow two-dimensional typical section behavior in plunge, pitch and control surface motions, and the tests were performed using a open-circuit, blower-type low-speed wind tunnel. Bifurcation analysis comparing both numerical and experimental results was carried out. Higher-order spectral (HOS) analysis was performed to investigate the quadratic and cubic forms of nonlinear couplings due to the combined hardening and free-play effects.

The paper content includes a description of the mathematical model and the structural nonlinear representations, followed by the theoretical aspects of higher-order spectra analysis. A description of the experimental apparatus and test set-up is presented, where the main parameters used for numerical simulations are also presented. Then, bifurcation analysis results and higher-order spectra investigation for couplings are discussed and concluding remarks are presented.

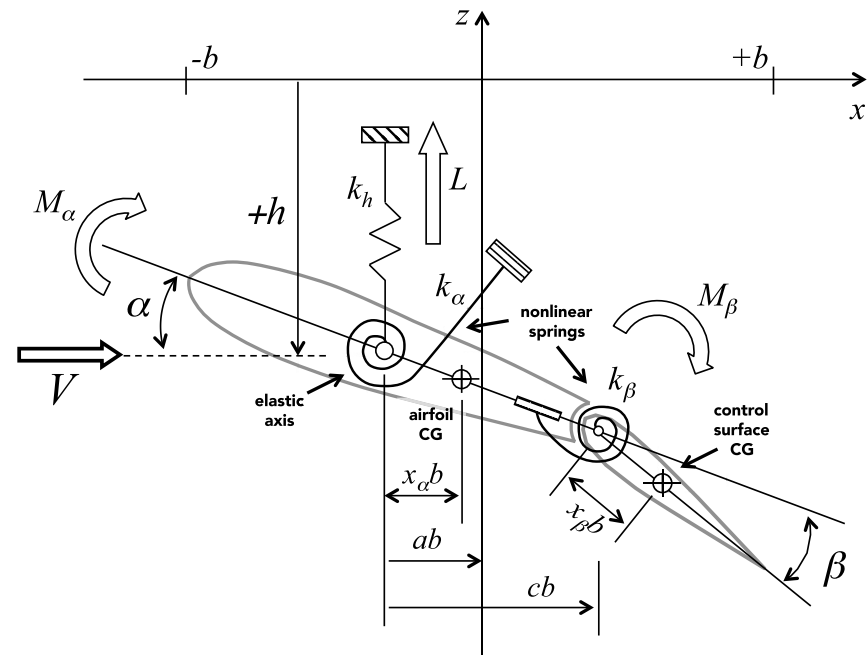

Fig. 2. Typical aeroelastic section representation.

\section{Mathematical model}

\subsection{Aeroelastic equations}

The mathematical model for typical section with three-degree of freedom ( $c f$. Fig. 2) is derived admitting the basic principles given in Refs. [1,13], and the formulation is detailed in Ref. [7]. The resulting set of aeroelastic equations is,

$$
\begin{aligned}
& {\left[\begin{array}{ccc}
\left(\frac{m_{T}}{m_{W}}\right) & x_{\alpha} & x_{\beta} \\
x_{\alpha} & r_{\alpha}^{2} & {\left[r_{\beta}^{2}+(c-a) x_{\beta}\right]} \\
x_{\beta} & {\left[r_{\beta}{ }^{2}+(c-a) x_{\beta}\right]} & r_{\beta}{ }^{2}
\end{array}\right]\left\{\begin{array}{c}
\ddot{\xi}(t) \\
\ddot{\alpha}(t) \\
\ddot{\beta}(t)
\end{array}\right\}} \\
& +\left[\begin{array}{lll}
d_{1,1} & d_{1,2} & d_{1,3} \\
d_{2,1} & d_{2,2} & d_{2,3} \\
d_{3,1} & d_{3,2} & d_{3,3}
\end{array}\right]\left\{\begin{array}{c}
\dot{\xi}(t) \\
\dot{\alpha}(t) \\
\dot{\beta}(t)
\end{array}\right\}+ \\
& +\left[\begin{array}{ccc}
\omega_{h}^{2} & 0 & 0 \\
0 & r_{\alpha}^{2} \omega_{\alpha}^{2} \frac{F(\alpha)}{\alpha(t)} & 0 \\
0 & 0 & r_{\beta}^{2} \omega_{\beta}^{2} \frac{F(\beta)}{\beta(t)}
\end{array}\right]\left\{\begin{array}{c}
\xi(t) \\
\alpha(t) \\
\beta(t)
\end{array}\right\} \\
& =\left(\frac{1}{b^{2} m_{W}}\right)\left\{\begin{array}{c}
-b L(t) \\
M_{\alpha}(t) \\
M_{\beta}(t)
\end{array}\right\},
\end{aligned}
$$

where $b$ is the semi-chord of the airfoil, $U$ is the airspeed, $h(t)$, $\alpha(t)$, and $\beta(t)$ are the plunge (positive downwards), pitch and control surface (trailing edge movable tab) displacements, respectively, $\xi(t)=\frac{h(t)}{b}$ is the non-dimensional plunge displacement, $a$ is the distance in $x$-direction of the elastic axis position from the reference system origin in proportion of the airfoil semi-chord, $c$ is the distance in $x$-direction of the control surface hinge position from the reference system origin also proportional to the airfoil semi-chord, $x_{\alpha}$ and $x_{\beta}$ are the dimensionless distances from elastic axis respectively to the airfoil and the control surface centers of gravity (CG), $r_{\alpha}$ and $r_{\beta}$ are the airfoil (with respect to the elastic axis) and control surface (with respect to the hinge line) radius of gyration, respectively, $k_{h}, k_{\alpha}$, and $k_{\beta}$ are the plunge, pitch, and control surface stiffness values, respectively, $m_{W}$ is the wing (airfoil) weight, $m_{T}$ is the total weight of the aeroelastic device considering all moving masses not immersed in the flowfield, $d_{i, j}$ are added structural damping factors with respect to each airfoil motion (Rayleigh approach), $L(t)$ is the unsteady lift force, $M_{\alpha}(t)$ and $M_{\beta}(t)$ are the unsteady pitch and hinge aerodynamic moments, respectively, $F(\alpha)$ and $F(\beta)$ are functions representing the nonlinearities related to pitch and control surface motions, respectively. 


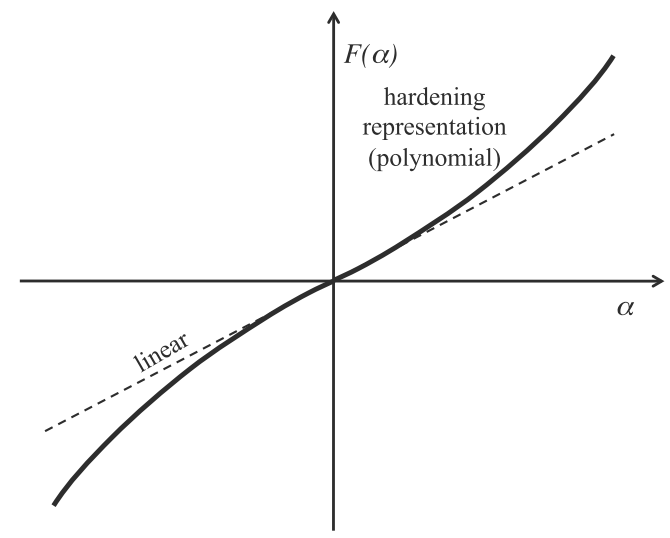

(a) Hardening representation

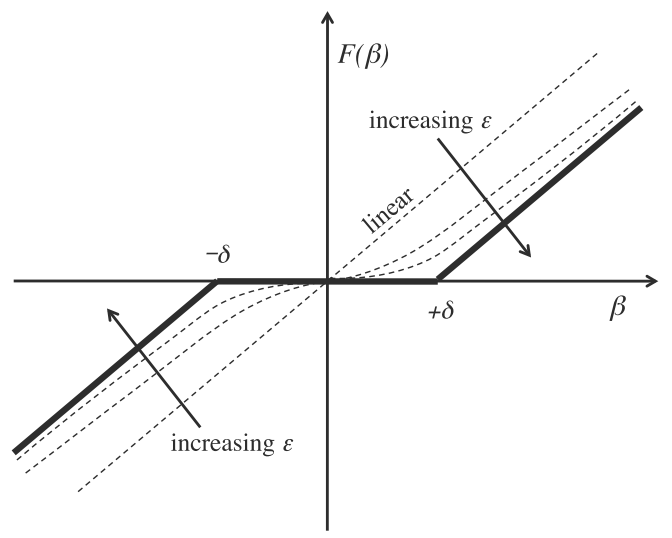

(b) Free-play representation

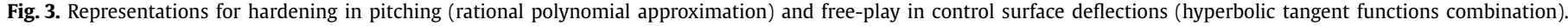

Unsteady aerodynamic loads modeling is based on the generalized Theodorsen formulation [13], in which the convolution of Wagner function is used to expand the Theodorsen function (harmonic motions) in order to account for arbitrary airfoil motions. Details on this approach can be obtained from Ref. [7,11], as well as the final aeroelastic set of equations given in the state space form, that is,

$\dot{\mathbf{x}}(t)=\mathbf{A}(\mathbf{x}(t)) \mathbf{x}(t)$

where, $\mathbf{A}(\mathbf{x}(t))$ is the state matrix, $\mathbf{x}(t)=\left[\begin{array}{llll}\xi(t) & \alpha(t) & \beta(t) & \dot{\xi}(t)\end{array}\right.$ $\left.\begin{array}{llll}\dot{\alpha}(t) & \dot{\beta}(t) & \bar{x}_{a}(t) & \dot{\bar{x}}_{a}(t)\end{array}\right]^{\mathrm{T}}$ is the state vector, and $\bar{x}_{a}(t)$ is an added aerodynamic state.

\subsection{Nonlinear representation}

Functions $F(\alpha)$ and $F(\beta)$ in Eq. (1) are used to feed that aeroelastic set with the respective terms to account for nonlinearities in pitch and control surface responses. Here the hardening and free-play effects are combined for those motions ( $c f$. Fig. 3), being: (i) for pitching motion only hardening nonlinearity is considered, while, (ii) free-play is only affecting the control surface motion.

The hardening nonlinearity function in pitching $(F(\alpha))$ has been obtained using the rational polynomials approximation (RP) [14] from the experimentally measured restoring pitching moment curve (cf. Fig. 3(a)). The RP approximation approach leads to the following ratio of polynomials form,

$F(\alpha)=\frac{a_{3} \alpha^{3}+a_{2} \alpha^{2}+a_{1} \alpha+a_{0}}{b_{2} \alpha^{2}+b_{1} \alpha+b_{0}}$,

where $a_{0}$ to $a_{3}$, and $b_{0}$ to $b_{2}$ are real-valued coefficients obtained numerically from measured experimental data.

For the free-play nonlinearity representation in the restoring torque of control surface, hyperbolic tangent functions combination is used as proposed and validated by Vasconcellos et al. [11]. This function is given by:

$$
\begin{aligned}
F(\beta)= & \frac{1}{2}[1-\tanh (\varepsilon(\beta+\delta))](\beta+\delta) \\
& +\frac{1}{2}[1+\tanh (\varepsilon(\beta-\delta))](\beta-\delta),
\end{aligned}
$$

where $\delta$ denotes the lower and the upper freeplay boundaries, and $\varepsilon$ is a variable which affects the smoothness of the function, where the higher is $\varepsilon$ the free-play discontinuity is better represented (cf. Fig. 3(b)).

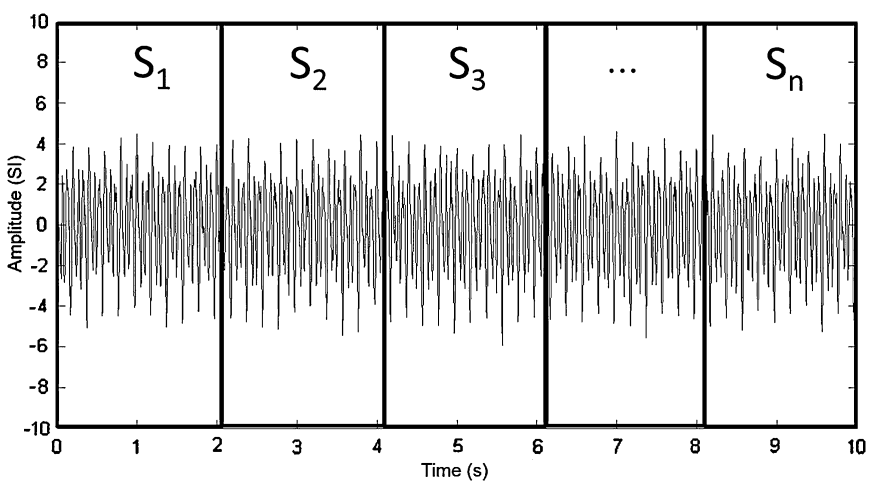

Fig. 4. Time series divided into $S_{n}$ segments.

\subsection{Higher-order spectra}

Higher-order spectra (HOS) moments are Fourier transforms of higher-order correlation functions that can be used to obtain more information about nonlinear coupling. This section presents the basic background of HOS analysis based on Refs. [12,15-17]. The Fourier or frequency spectra is an important tool that can help to identify and differentiate features of nonlinear systems related to stationary signals such as limit cycle oscillations and chaotic motions. The Fourier transform of a signal $x(t)$ is defined as

$X(f)=\int_{-\infty}^{\infty} x(t) e^{-2 i \pi f t} d t$

where $f$ denotes the frequency and $X(f)$ is a complex quantity.

The power spectrum $\left(S_{x x}\right)$ for a discretely sampled data is obtained from

$S_{X X}(f)=\lim _{T \rightarrow \infty} \frac{1}{T} E\left[X(f) X^{*}(f)\right]$,

where $X(f)$ is the Fourier transform (amplitude spectrum), $E[\cdot]$ denotes the expected value calculated by the arithmetic average estimator, and the superscript $\left(^{*}\right)$ denotes complex conjugate. (See Fig. 4.) from

The auto-bispectrum and auto-trispectrum are similarly obtained

$S_{x x x}\left(f_{1}, f_{2}\right)=\lim _{T \rightarrow \infty}\left(\frac{1}{T}\right) E\left[X\left(f_{1}+f_{2}\right) X^{*}\left(f_{1}\right) X^{*}\left(f_{2}\right)\right]$,

$S_{x x x x}\left(f_{1}, f_{2}, f_{3}\right)$

$=\lim _{T \rightarrow \infty}\left(\frac{1}{T}\right) E\left[X\left(f_{1}+f_{2}+f_{3}\right) X^{*}\left(f_{1}\right) X^{*}\left(f_{2}\right) X^{*}\left(f_{3}\right)\right]$. 


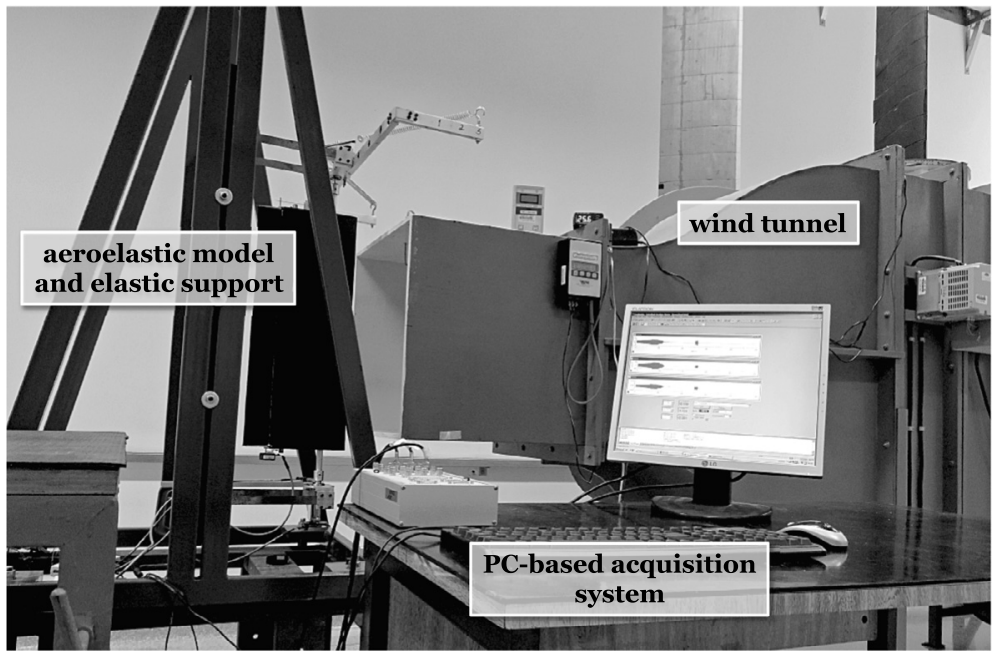

Fig. 5. Experimental apparatus.

In nonlinear systems, frequency components can interact to form new components at their sum or difference frequency [15]. When interacting the phase of the new component is related to the phases of the primary interacting modes, therefore by inspecting the phase relation it is possible to identify any nonlinear coupling.

The basis of HOS analysis in detecting nonlinear couplings among frequency modes relies on the fact that quadratic interaction of two frequency components $\left(f_{1}\right.$ and $f_{2}$ ) and their sum components $\left(f_{1}+f_{2}\right)$ leads to large values for auto-bispectrum. Similarly, the cubic interaction of three frequency components $\left(f_{1}\right.$, $f_{2}$, and $f_{3}$ ) yields a large value for the auto-trispectrum.

The auto-bispectrum and auto-trispectrum are usually normalized with respect to the amplitudes of the individual spectral components to yield the auto-bicoherence and auto-tricoherence. Therefore, based on the Schwartz inequality, are defined respectively as,

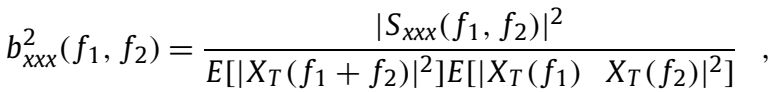

$$
\begin{aligned}
& t_{x x x x}^{2}\left(f_{1}, f_{2}, f_{3}\right)
\end{aligned}
$$

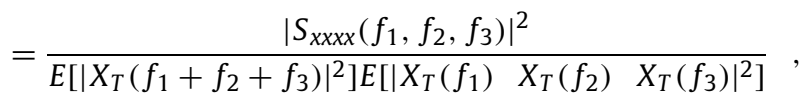

where $0<b_{x x x}^{2}\left(f_{1}, f_{2}\right)<1$ and $0<t_{x x x x}^{2}\left(f_{1}, f_{2}, f_{3}\right)<1$.

If $b_{x x x}^{2}\left(f_{1}, f_{2}\right)=1$, then the pair of frequency components at $f_{1}$ and $f_{2}$, as well as their sum $f_{1}+f_{2}$, are quadratically coupled. If $b_{x x x}^{2}\left(f_{1}, f_{2}\right)=0$, frequency components are not coupled, and partially coupled if $0<b_{x x x}^{2}\left(f_{1}, f_{2}\right)<1$. A two dimensional plot of cut-off planes from auto-bicoherence can reveal regions in which the frequencies are coupled. Similarly, a unit value of auto-tricoherence indicates perfect cubic phase coupling, zero values indicates no coupling, and values between zero and one indicates partial coupling. In this case, to observe the coupling levels it is necessary to plot a three dimensional surface representing a tridimensional cut-off region. Table 1 shows a summary on HOS analysis and possible nonlinear interactions.

\section{Experimental set-up and parameters}

The aeroelastic tests were performed with a rigid wing (span of $0.8 \mathrm{~m}$ ) fixed to an elastic suspension that allows analogy to a three-degree of freedom typical airfoil section (plunge, pitch, and control surface). Fig. 5 depicts the experimental apparatus prepared to be tested in the open-section blower-type wind tunnel $(500 \times 500 \mathrm{~mm})$.
Table 1

Higher order spectral analysis technique.

\begin{tabular}{lll}
\hline & \multicolumn{2}{c}{ NONLINEAR INTERACTION } \\
\hline Auto-bicoherence & $b_{x x x}^{2}\left(f_{1}, f_{2}\right)=1$ & Quadratic phase coupling \\
& $0<b_{x x x}^{2}\left(f_{1}, f_{2}\right)<1$ & Partial quadratic coupling \\
& $b_{x x x}^{2}\left(f_{1}, f_{2}\right)=0$ & No quadratic coupling \\
Auto-tricoherence & $t_{x x x x}^{2}\left(f_{1}, f_{2}, f_{3}\right)=1$ & Cubic phase coupling \\
& $0<t_{x x x x}^{2}\left(f_{1}, f_{2}, f_{3}\right)<1$ & Partial cubic coupling \\
& $t_{x x x x}^{2}\left(f_{1}, f_{2}, f_{3}\right)=0$ & No cubic coupling \\
\hline
\end{tabular}

Fig. 6 shows a sketch of the aeroelastic system. It is observed that the plunge motion is restrained by four elastic steel beams (two on the top and two on the bottom). Those beams also are responsible for connecting the model to a support frame appropriately located in front of the wind tunnel flow. The pitch stiffness is set by the two springs connected by a support device and each one in the opposite side of a nonlinear cam. This assembly is depicted in the photos presented in Fig. 7. The nonlinear cam was designed to allow for varying the intensity of the hardening nonlinearity. Control surface stiffness is provided by a piano wire and free-play adjustment gear, as depicted in Fig. 8. The measurements of the three-degree of freedom are done using encoders (angular and linear ones). The $\mathrm{dSPACE}^{\circledR}$ DS1104 R\&D Controller Board system together with Simulink ${ }^{\circledR}$ were used in the signal acquisition and processing.

In the numerical simulations, uncoupled frequencies were used. The uncoupled plunge, pitch, and control surface frequencies were assessed by individually restraining two motions and performing the modal test for the other motion. A summary of the aeroelastic model parameters assumed in the numerical simulation is given in Table 2.

Structural damping is an important quantity and impacts the system's response. Here, the damping matrix in Eq. (1) is accounted for by using the Rayleigh approach,

$d_{i, j}=a_{0} m_{i, j}+a_{1} k_{i, j}$,

where $m_{i, j}$ and $k_{i, j}$ are the mass and stiffness matrices elements (cf. Eq. (1)) before nonlinear deformations occur, respectively, and factors $a_{0}$ and $a_{1}$ are the so-called Rayleigh factors.

The Rayleigh factors can be evaluated by the solution of a pair of simultaneous equations if the damping ratios $\zeta_{m}$ and $\zeta_{n}$ associated with two specific frequencies (modes) $\omega_{m}$ and $\omega_{n}$ are known, that is, 


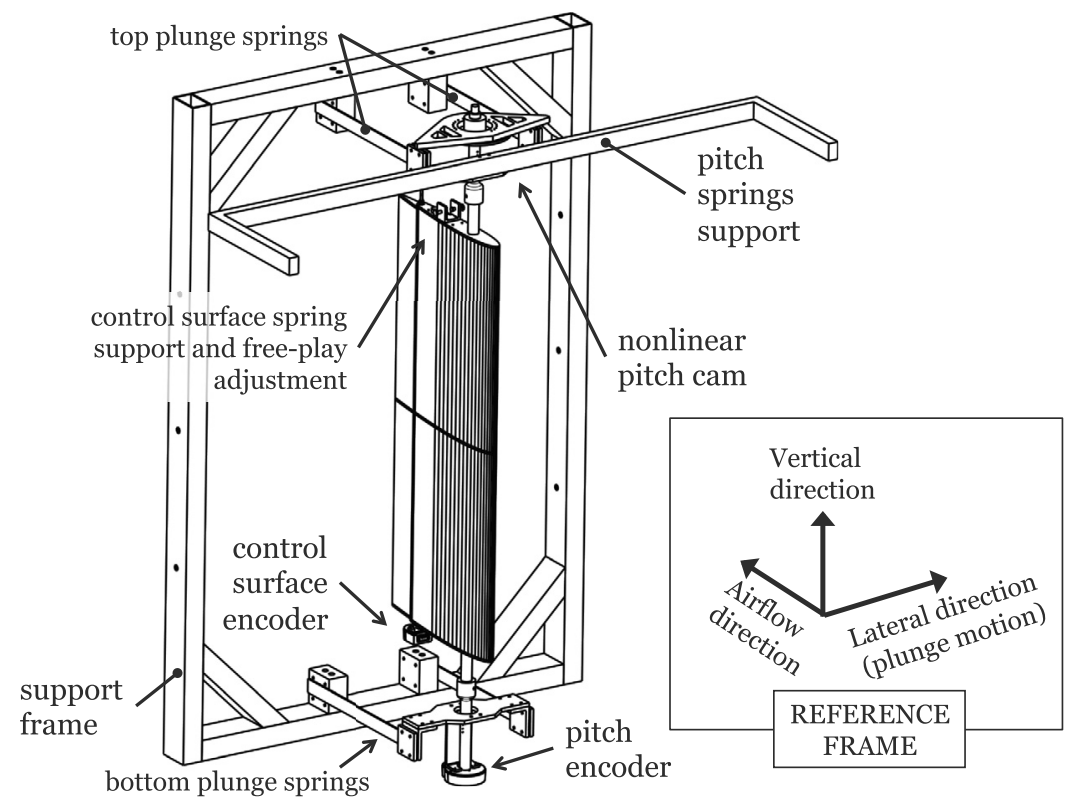

Fig. 6. Sketch of the experimental model

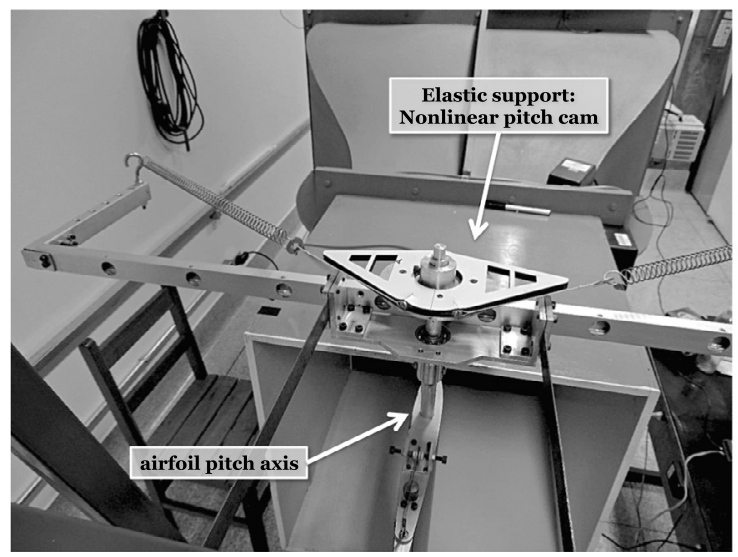

(a) Top/back view

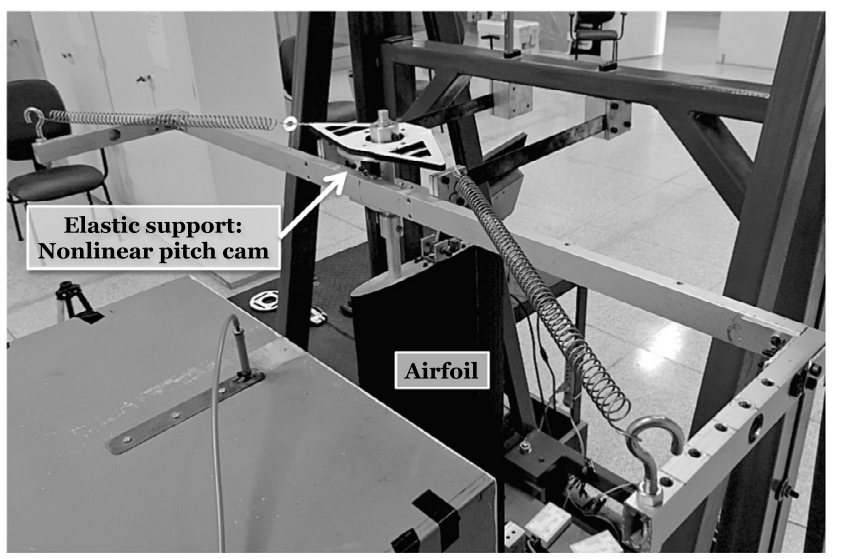

(b) Side view

Fig. 7. Details of the nonlinear pitch cam assemble.

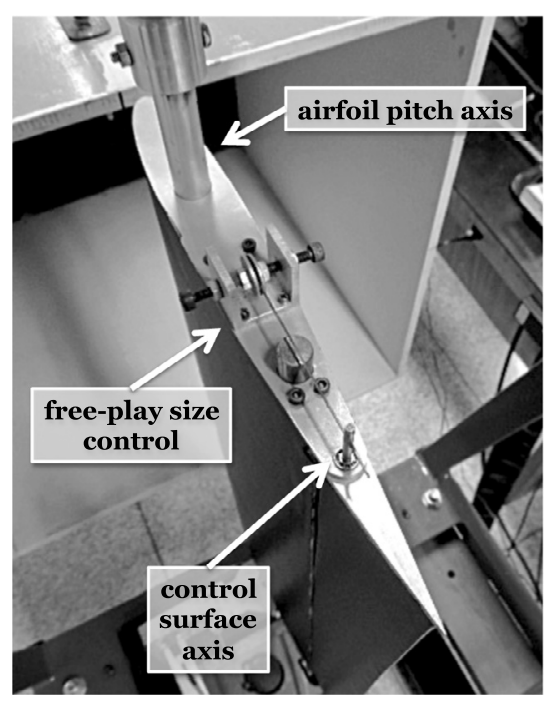

Fig. 8. Details of the control surface free-play adjustment device.

$$
\left\{\begin{array}{l}
a_{0} \\
a_{1}
\end{array}\right\}=2 \frac{\omega_{m} \omega_{n}}{\omega_{n}^{2}-\omega_{m}^{2}}\left[\begin{array}{cc}
\omega_{n} & -\omega_{m} \\
\frac{-1}{\omega_{n}} & \frac{1}{\omega_{m}}
\end{array}\right]\left\{\begin{array}{c}
\zeta_{m} \\
\zeta_{n}
\end{array}\right\} .
$$

The damping ratios in Table 2 were used to evaluate $d_{i, j}$ with Eq. (11). Here, the following ratios were admitted: $\zeta_{m}=\zeta_{\alpha}, \zeta_{n}=$ $\zeta_{\beta}, \omega_{m}=\omega_{\alpha}$, and $\omega_{n}=\omega_{\beta}$. Aeroelastic experiments and numerical simulations were performed considering hardening nonlinearity in pitching and free-play nonlinearity in the control surface hinge. Combinations of the nonlinearities were admitted and based in three different intensities of hardening and two sizes for the freeplay gaps.

The three intensities of the hardening nonlinearity in pitching were obtained by adjusting the pitch spring sizes using the respective support as illustrated in Figs. 6 and 7. The hardening effects vary from large, medium, and small increasing in restoring pitching moment, denoted as hardening 3, 2, and 1, respectively. The restoring moment curve with respect to the pitch angle was assessed and the rational polynomial approximation was used to fit these curves. Fig. 9 presents both experimentally acquired restoring moment curves for the different hardening, as well as the respective polynomial fittings. Table 3 presents the respective RP approximation coefficients for each hardening intensity. 
Table 2

Experimental values used for the numerical model.

\begin{tabular}{lll}
\hline VARIABLES & DESCRIPTION & VALUES \\
\hline $\mathrm{b}$ & Semi-chord $(\mathrm{m})$ & 0.125 \\
$\mathrm{a}$ & Distance from semi-chord to elastic axis (nondimensional) & -0.5 \\
$\mathrm{c}$ & Hinge line location measured from mid-chord (nondimensional) & 0.5 \\
$\rho$ & Air density $\left(\mathrm{kg} / \mathrm{m}^{3}\right)$ & 1.078 \\
$m_{W}$ & Wing mass $(\mathrm{kg})$ & 1.5 \\
$m_{T}$ & Total mass $(\mathrm{kg})$ & 4.3723 \\
$\omega_{h}$ & Decoupled plunge natural frequency (rad/s) & 27.3268 \\
$\omega_{\alpha}$ & Decoupled pitch natural frequency (rad/s) & 12.11 \\
$\omega_{\beta}$ & Decoupled control surface natural frequency (rad/s) & 50.2761 \\
$x_{\alpha}$ & Nondimensional distance between elastic axis and CG of wing & 0.66 \\
$x_{\beta}$ & Nondimensional distance between hinge line and CG of flap & 0.0028 \\
$r_{\alpha}$ & Nondimensional rotational inertia term about elastic axis & 0.7303 \\
$r_{\beta}$ & Nondimensional rotational inertia term about hinge line & 0.0742 \\
$\mu$ & Nondimensional mass ratio & 28.3467 \\
$\zeta_{h}$ & Plunge modal damping ratio & 0.1275 \\
$\zeta_{\alpha}$ & Pitch modal damping ratio & 0.3697 \\
$\zeta_{\beta}$ & Flap modal damping ratio & 0.0106 \\
$U_{f}$ & Linear flutter velocity (Numeric) (m/s) & 11.465 \\
$U_{f}^{*}$ & Critical flutter velocity (Experiment) (m/s) & $12.0 \leq U_{f}^{*} \leq 12.20$ \\
\hline
\end{tabular}

Table 3

Rational polynomial coefficients for three different intensities of hardening nonlinearities.

\begin{tabular}{|c|c|c|c|c|c|c|c|}
\hline & $a_{3}$ & $a_{2}$ & $a_{1}$ & $a_{0}$ & $b_{2}$ & $b_{1}$ & $b_{0}$ \\
\hline Hardening 3 & 7.281 & $3.01 \times 10^{-2}$ & $1.33 \times 10^{-2}$ & $-1.44 \times 10^{-4}$ & 1.0 & $6.39 \times 10^{-3}$ & $1.91 \times 10^{-2}$ \\
\hline Hardening 2 & 6.313 & $-4.58 \times 10^{-2}$ & $3.64 \times 10^{-2}$ & $-2.48 \times 10^{-4}$ & 1.0 & $-1.06 \times 10^{-2}$ & $2.54 \times 10^{-2}$ \\
\hline Hardening 1 & 6.403 & $-4.76 \times 10^{-3}$ & $1.26 \times 10^{-1}$ & $-3.03 \times 10^{-4}$ & 1.0 & $2.61 \times 10^{-7}$ & $6.37 \times 10^{-2}$ \\
\hline
\end{tabular}

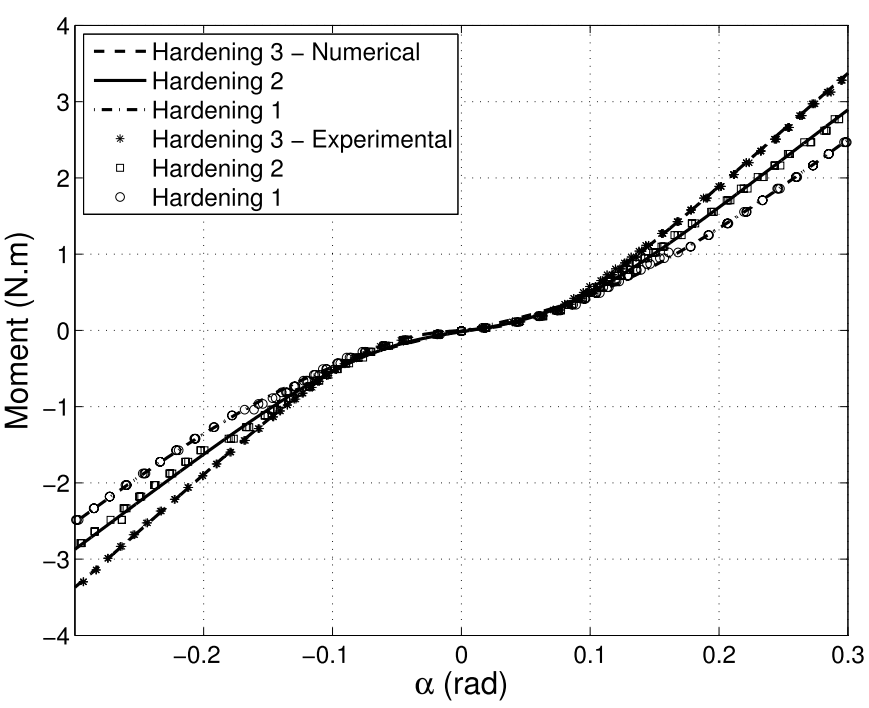

Fig. 9. Restoring pitch moment subjected to hardening effects of three intensities.

As far as free-play nonlinearity is concerned, two gap values were considered, that is, 2.0 and 4.0 degrees, and referred from now on as $2 \delta=2^{\circ}$ and $2 \delta=4^{\circ}$, respectively.

\section{Hopf bifurcation analysis}

Hopf bifurcation is a typical phenomenon in nonlinear aeroelastic systems, where limit cycle oscillations (LCOs) are observed at a particular airflow velocity. The bifurcation onset can be either observed experimentally or simulated numerically. Here, the numerically predicted linear flutter velocity is taken as the reference to compare the measured and numerically predicted limit cycle oscillations. To assess the linear flutter condition, the eigenvalues of the state matrix in Eq. (2) are inspected against the air velocity. The first eigenvalue presenting positive real part determines the critical flutter velocity. The numerical flutter velocity, $U_{f}$, is $11.465 \mathrm{~m} / \mathrm{s}$ and the experimental flutter velocity, $U_{f}^{*}$, was approximated to be in the interval $12.0<U_{f}^{*}<12.2 \mathrm{~m} / \mathrm{s}$, thereby representing an error in between 4.45 to $6.0 \%$. As such, we compare LCO characteristics at normalized velocities, which are obtained by dividing the numerical velocities by $U_{f}=11.465 \mathrm{~m} / \mathrm{s}$ and the experimental ones by $U_{f}^{*}=12.10 \mathrm{~m} / \mathrm{s}$. The normalized flutter velocity is then referred as the critical velocity, $U_{c}$.

\subsection{Influence of hardening nonlinearity}

Fig. 10 depicts the amplitudes of the limit cycle oscillations for each of the three-degree of freedom from the numerical simulations and experimental results for varying levels of nonlinear hardening in the pitch motion only. No free-play effect is included to this analysis. Three different hardening intensities were imposed as previously presented in Fig. 9 and Table 3.

The results reveal a subcritical behavior, where the system exhibits limit cycle oscillations at airspeeds that are smaller than the critical flutter speed. In the numerical results, limit cycle oscillations are observed at $U=0.945 U_{c}$ for all hardening curves. Near the onset of oscillations, the plunge amplitudes are approximately $0.4,0.45$, and $0.5 \mathrm{~cm}$ for the numeric hardening 3,2 , and 1 , respectively, indicating an increase in the plunge oscillation amplitude as the hardening nonlinearity is decreased. The amplitude of pitch and surface control motions for the bifurcation onset are $3.2^{\circ}, 3.6^{\circ}$, $3.9^{\circ}$, and $0.6^{\circ}, 0.68^{\circ}$, are $0.76^{\circ}$ for the hardening 3,2 and 1 , respectively. The amplitudes of these motions are also larger at the smaller nonlinear hardening effects. In the experimental measurements, the bifurcation onset is close to $0.97 U_{c}$, since the accuracy of the tunnel does not allow capturing the precise instability onset point. Similar variations in the amplitudes of all motions are noted as the level of hardening nonlinearity is enhanced.

Fig. 11 shows comparisons of the measured and simulated time series and calculated power spectra for the hardening 3 case at $U=1.18 U_{c}$. The plots show good agreement between the experimental and numerical results for all degrees of freedom (plunge, pitch, and control surface deflection). From power spectra, it is 


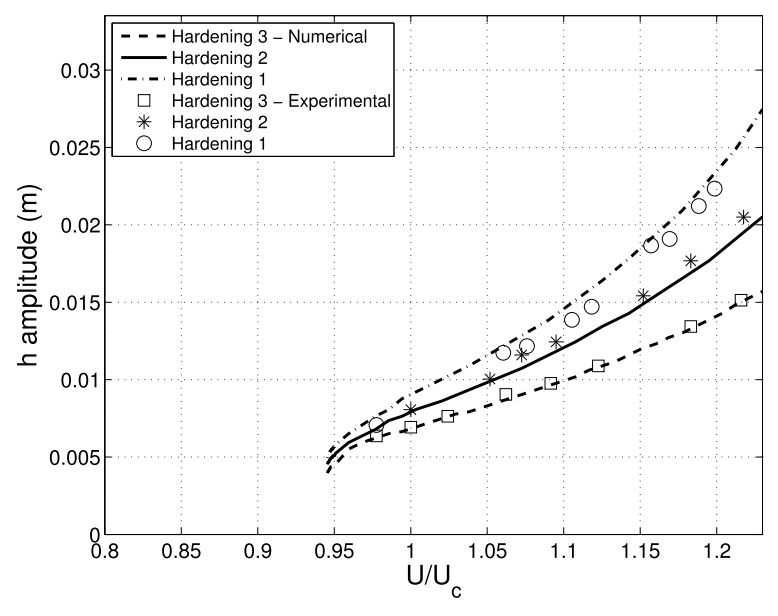

(a) Plunge

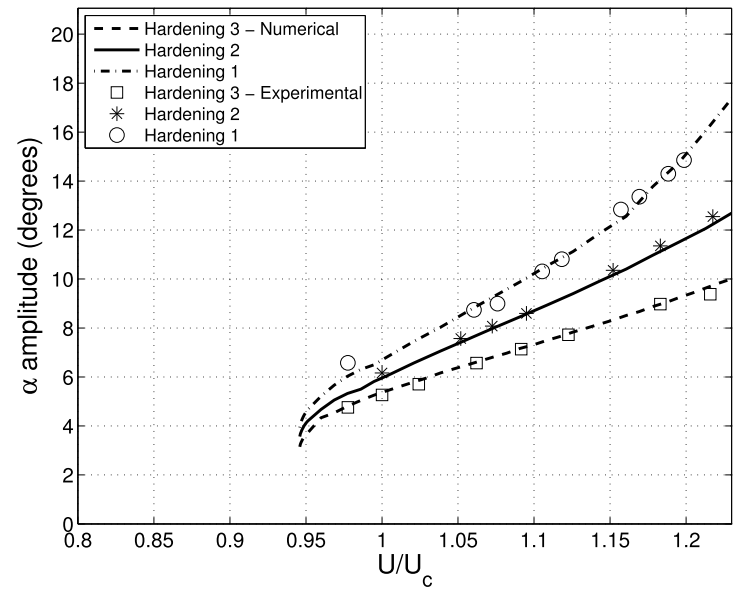

(b) Pitch

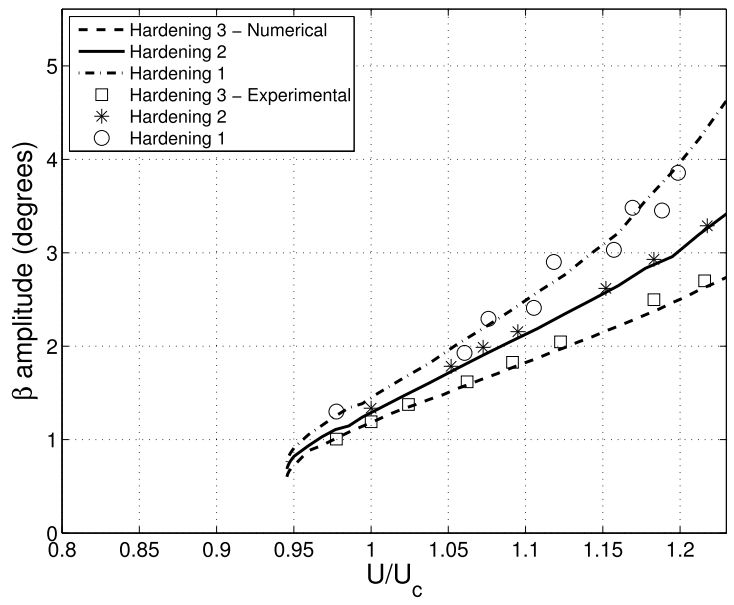

(c) Control surface

Fig. 10. Bifurcation diagrams for numerical and experimental results for hardening nonlinearities at different velocities.

observed that the experimental and numerical results yield the fundamental frequencies $\Omega_{\text {exp }}=2.87 \mathrm{~Hz}$ and $\Omega_{\text {num }}=2.93 \mathrm{~Hz}$ for the oscillations. It is also possible to observe that the numerical model captures both even and odd $(2 \Omega, 3 \Omega, 4 \Omega, 5 \Omega, \ldots)$ superharmonics that result from the hardening nonlinearity influence and some asymmetry in the pitch stiffness.

In general, the numerical model is validated, showing good agreement, as far the amplitude and the frequency of the oscillations of all degrees of freedom under different free stream velocities.

\subsection{Influence of free-play nonlinearity}

Fig. 12 depicts the aeroelastic system oscillations amplitudes of each d.o.f. for the numerical simulations and experimental results with combined hardening and free-play nonlinearities. The hardening 3 case is considered and two different free-play sizes $2 \delta=2^{\circ}$ and $2 \delta=4^{\circ}$ were assumed in these experiments and simulations. Subcritical bifurcations were observed in all cases. Although the numerical simulations show the effect of free-play nonlinearity increasing the unstable region, one could make the following observations. First, it is clear that increasing the gap size results in a decrease in the onset of LCO. This can best be observed in Fig. 12(c). Furthermore, the increase in gap increases the oscillation amplitudes of the control surface significantly. On the other hand, its effect on the amplitudes of the pitch and plunge motion are much smaller. The numerical simulations indicate the need for adjustments or, perhaps, adding extra effects on free-play modeling.

\section{HOS analysis}

To understand how the nonlinearities influence the aeroelastic system and to identify the sources of harmonics, HOS analysis was applied to the experimental results. The auto-spectrum was used to identify the frequency content of the system, while the autobispectrum was used to detect quadratic-like nonlinear behavior. Finally, the auto-trispectrum was used to assess the cubic-like nonlinear behavior, which is expected since hardening effect was induced to the device through its nonlinear pitch cam (cf. Fig. 7).

For the particular case of the apparatus to introduce hardening effect in the experiment, it is reasonable to conclude that it is unlikely to build a experimental device without symmetries. The auto-bicoherence confirms the phase coupling between the fundamental frequency and their even superharmonic frequencies, therefore used to verify typical quadratic-like nonlinear effect. Fig. 13 shows the auto-bicoherence contour plot and the power spectrum of the pitch motion experimental signals for the system under flutter velocity condition $\left(1.0 U_{c}\right)$. These results consider the system under the hardening 3 nonlinear curve, which presents some 


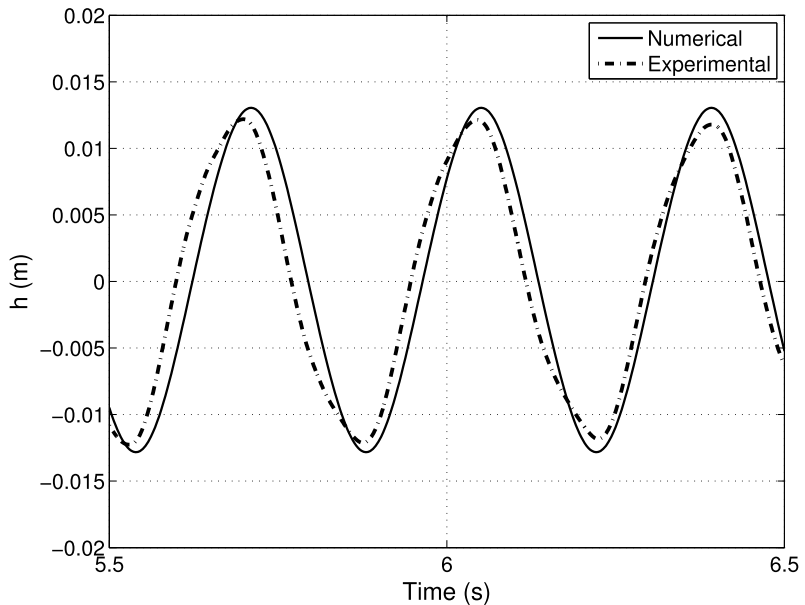

(a)

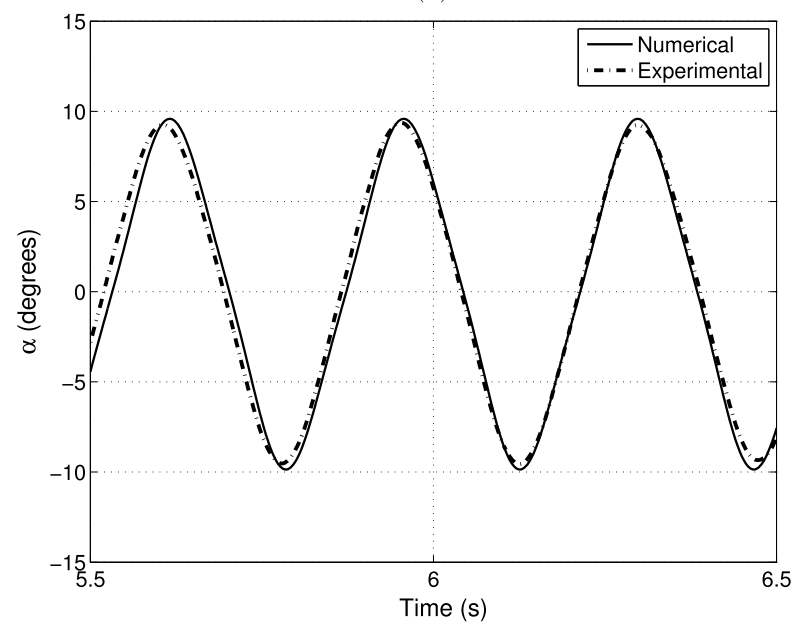

(c)

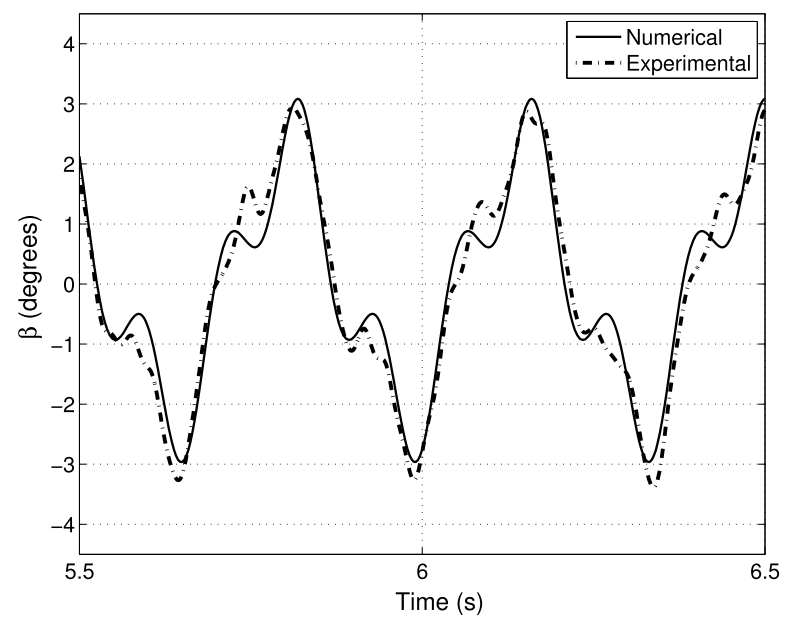

(e)

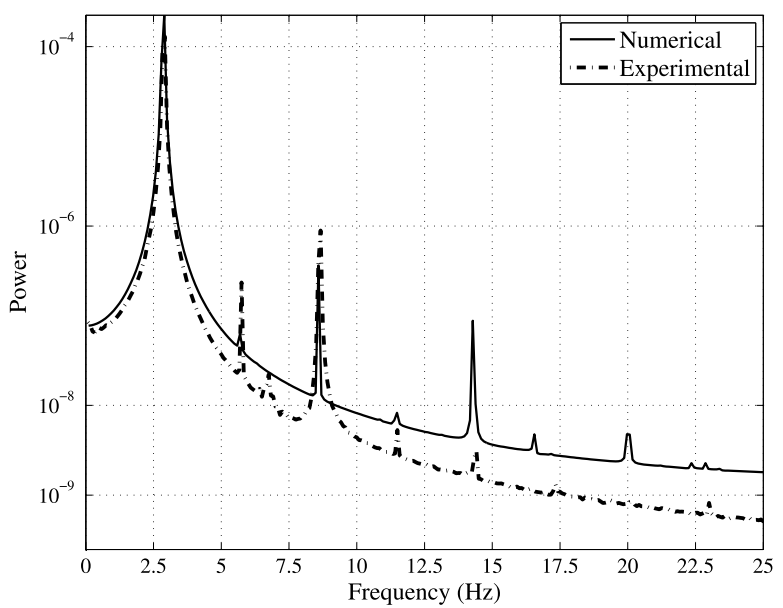

(b)

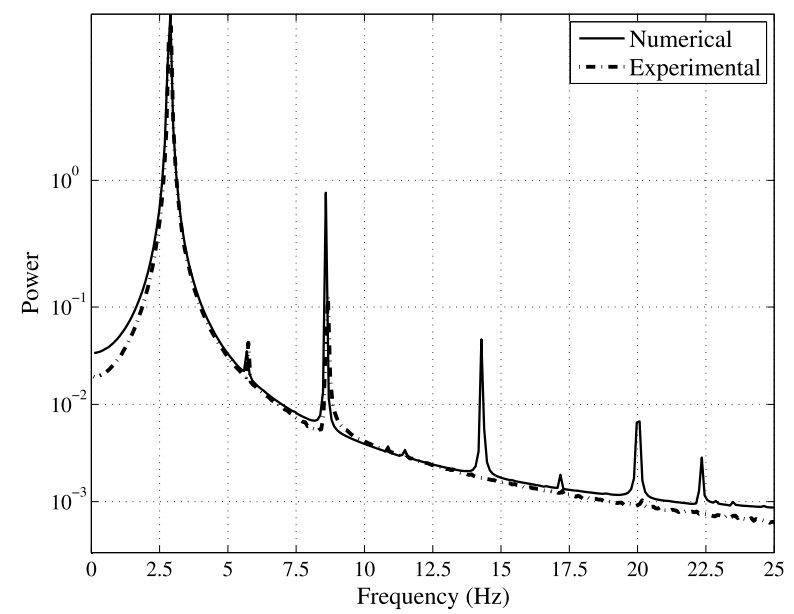

(d)

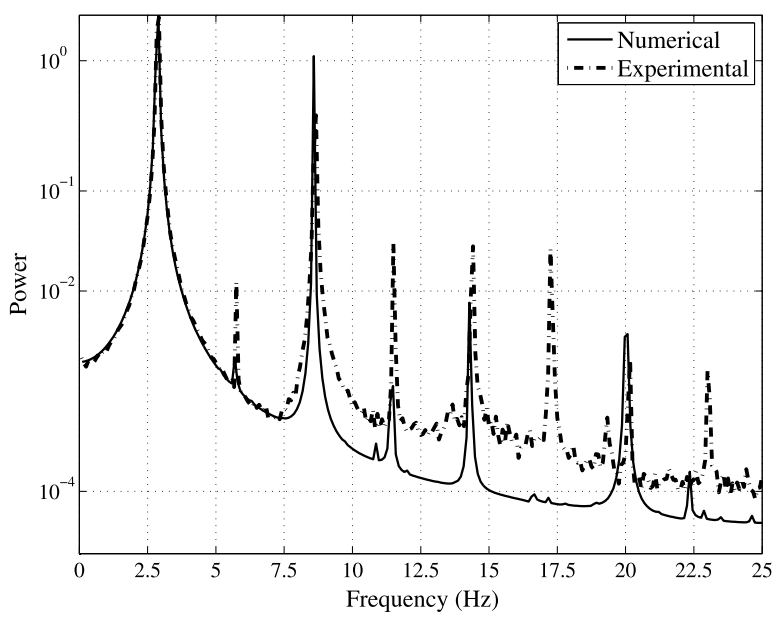

(f)

Fig. 11. Experimental and numerical time histories with respective power spectra of plunge, pitch and control surface motions (hardening 3 and airspeed $1.18 U_{c}$ ).

asymmetry. It is possible to see in the bicoherence contour plot the phase coupling between the fundamental harmonic with itself to generate the first $(2.73 \mathrm{~Hz}+2.73 \mathrm{~Hz}=5.46 \mathrm{~Hz})$ and other superharmonics. This system under this velocity also presents the subharmonic frequency $(0.7 \mathrm{~Hz}$ as in Fig. $13(\mathrm{~b}))$, which is coupled with the higher frequencies. The source of the quadratic coupling could be the admittance of a static position for the wing section or a small deformation in the pitch direction.
Fig. 14 shows the auto-tricoherence (contour level is set in 0.8 ) contour plot and the respective projections for the pitch motion experimental results, when the flow velocity is $1.0 U_{c}$ and $1.08 U_{c}$, respectively. The nonlinear cubic coupling between the fundamental frequency with itself generating the odd superharmonics, which was expected since the system is characterized by a hardening nonlinearity in the pitch. It is observed the interaction between the high frequencies increases as the airspeeds also 


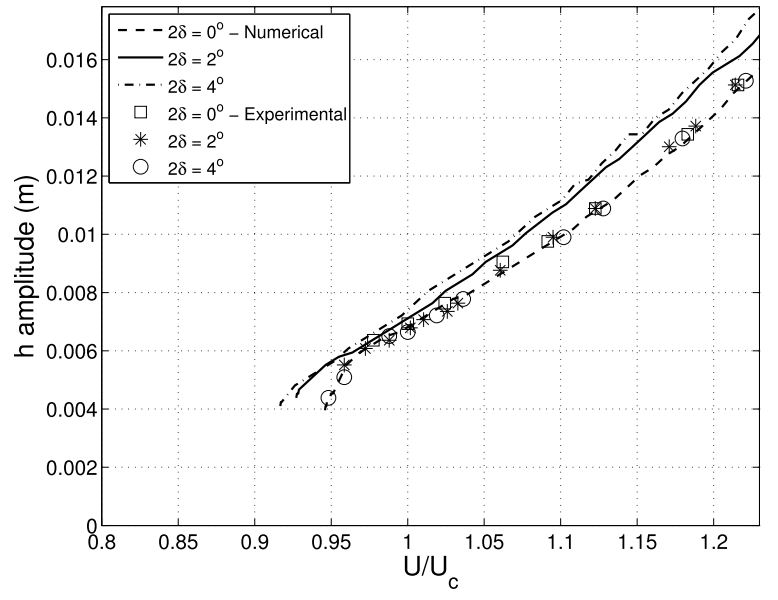

(a) Plunge

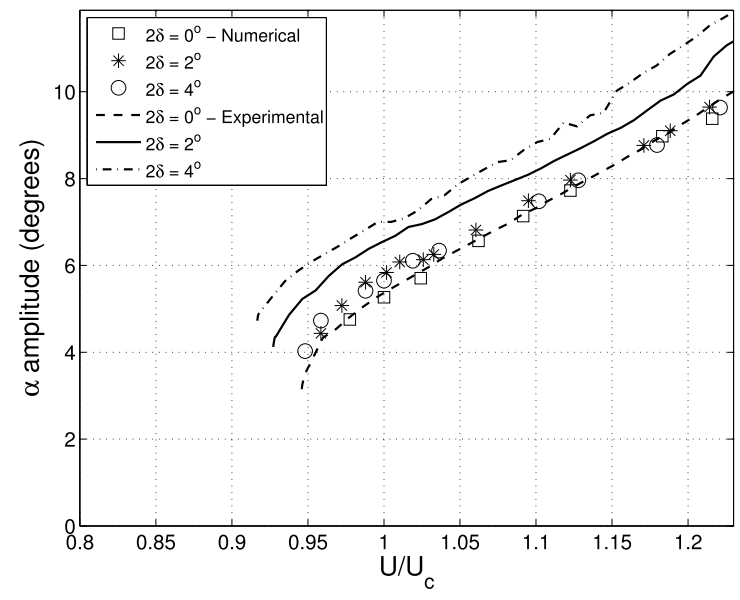

(b) Pitch

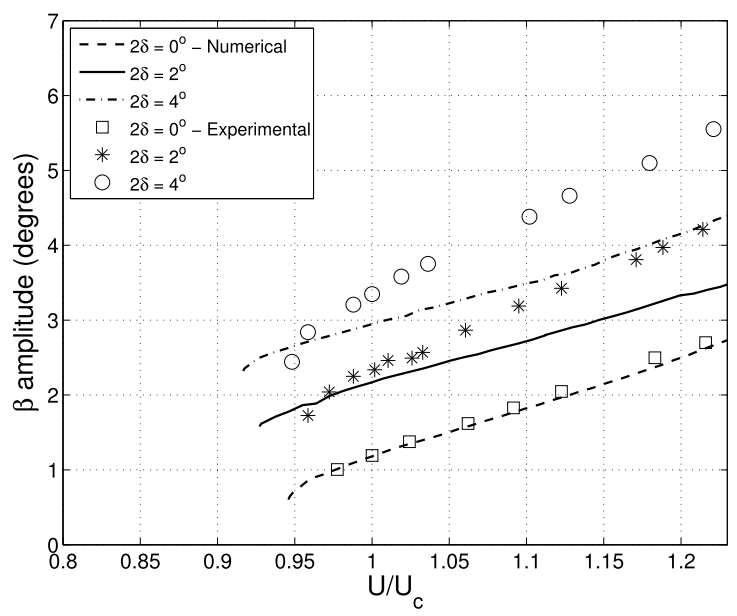

(c) Control surface

Fig. 12. Bifurcation diagrams for numerical and experimental results for free-play nonlinearities combined with hardening 3 nonlinearity in pitching at different velocities.

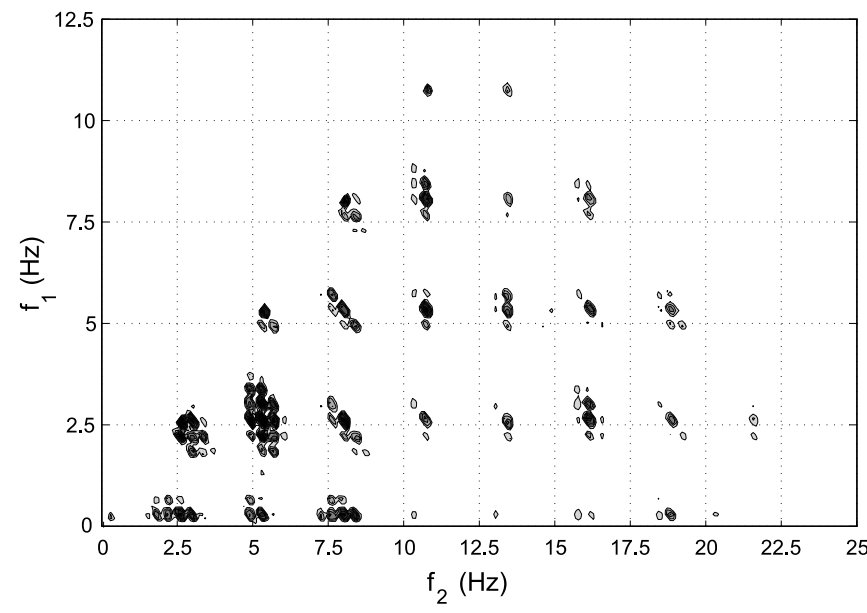

(a)

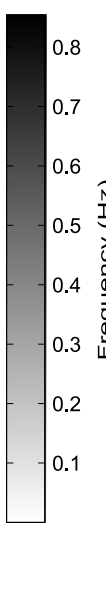

(1)

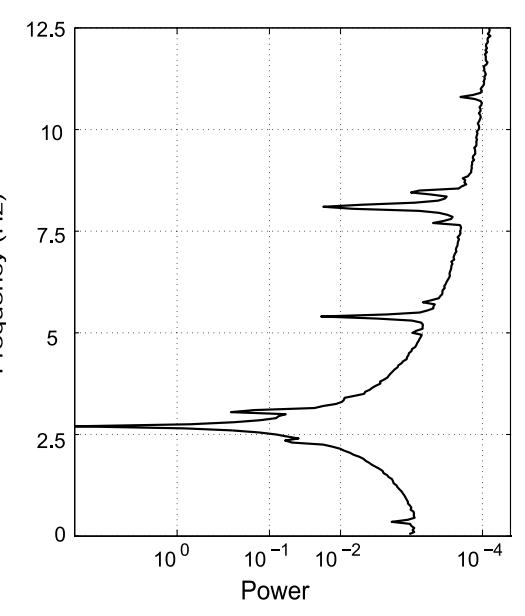

(b)

Fig. 13. Contour plot of the (a) autobicoherence (cut-off of 0.8 ) and (b) power spectrum density for the pitch motion when $1.0 U_{c}$.

increase (cf. Fig. 14). Moreover, the subharmonic frequency $(0.7 \mathrm{~Hz}$ in the $f_{2} \times f_{3}$ projection) disappears. This happens because of the strong energy transfer between the coupled frequencies for high airflow velocities, which increases the effect of the hardening nonlinearity.

\section{Concluding remarks}

This paper has presented a study on the influence of structural nonlinearities in a three-degree of freedom typical aeroelastic section through inspection of bifurcation diagrams and HOS analysis. 


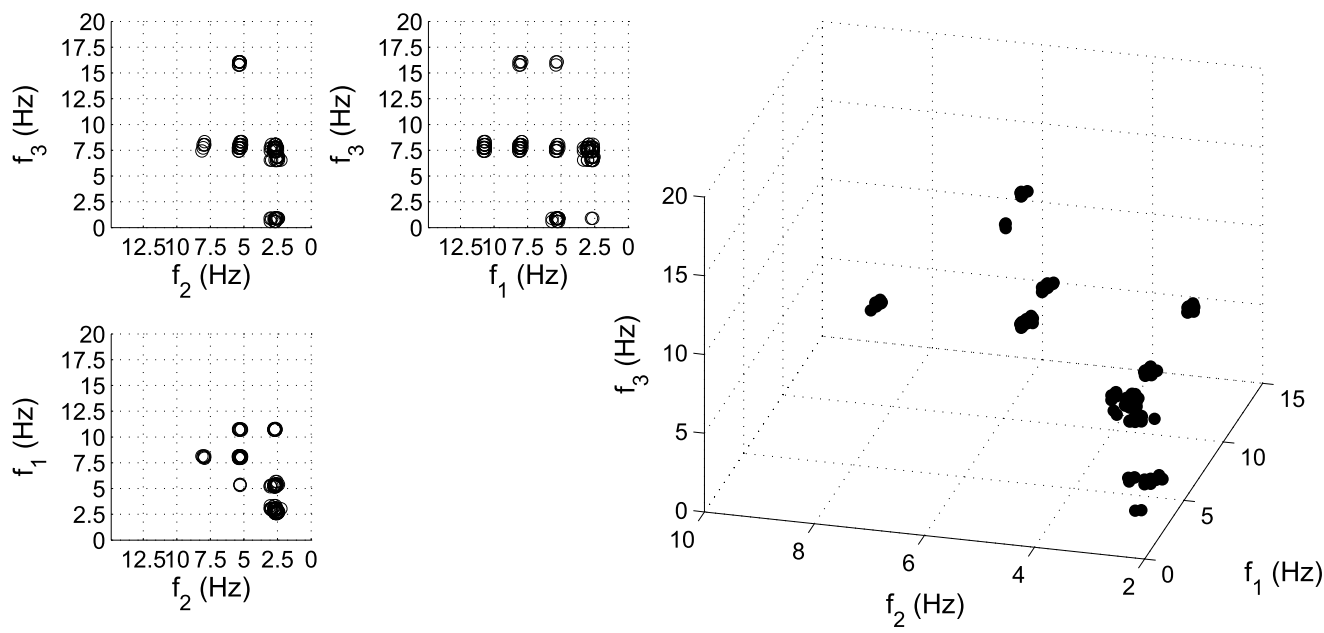

(a) $U=1.0 U_{c}$
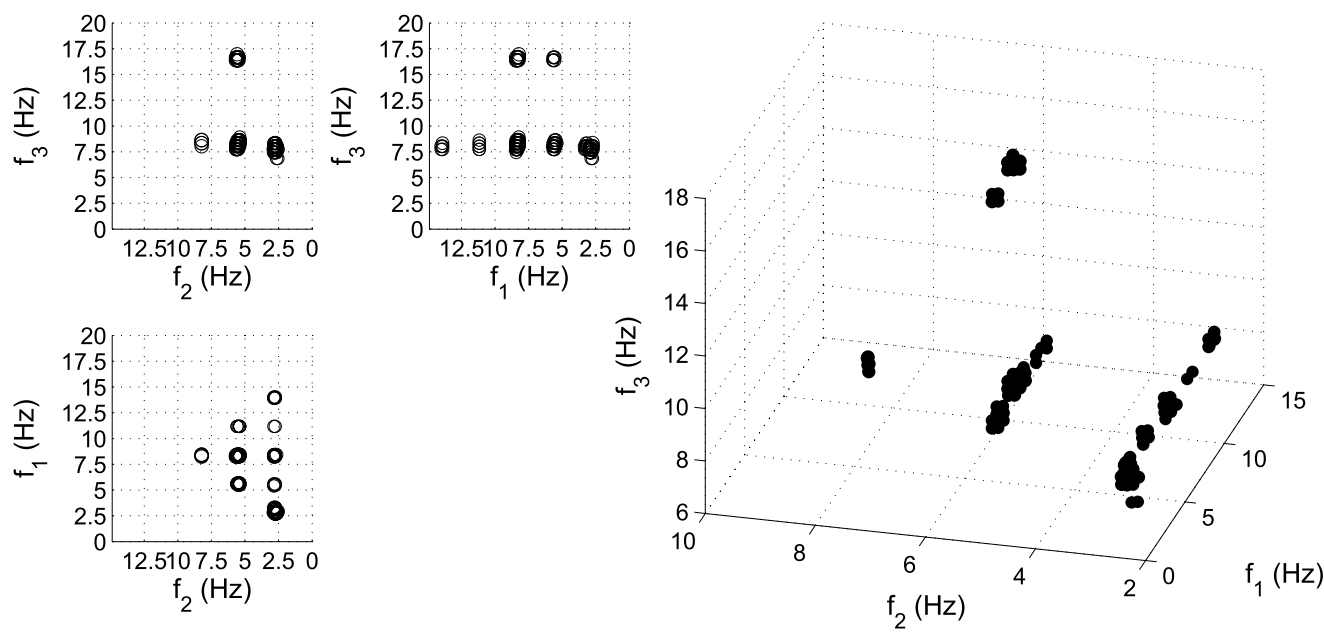

(b) $U=1.08 U_{c}$

Fig. 14. Auto-tricoherence surface contours and respective projections for the pitching motion in two airspeeds.

The numerical simulations and experimental results have focused on examining the combined effects of hardening in pitching motion and free-play in the control surface hinge on the system's response. As observed, the hardening nonlinearity is responsible for the appearance and amplitude level of LCOs. The free-play leads to the appearance of subcritical behavior and increases the region of dangerous LCO. In addition to that, it was observed that the linear parameters can play a role in determining the type of bifurcation instability. The HOS analysis confirmed the presence of quadratic and cubic nonlinearities and confirms the complexity on the system's response. Further work is planned to enhance the understanding of how different related nonlinearities can avoid the subcritical behavior through a proper parameter set.

\section{Conflict of interest statement}

The authors acknowledge that there is no conflict of interest of any sort.

\section{Acknowledgements}

The authors acknowledge the financial support from the Brazilian National Research Council - CNPq (Grant 305700/2013-8), and the Coordination for the Improvement of Higher Education Personnel (CAPES). They are also thankful to CAPES, CNPq, and FAPEMIG for funding this present research work through the INCT/EIE (CNPq 574001/2008-5).

\section{References}

[1] R.L. Bisphinghoff, H. Ashley, R.L. Halfman, Aeroelasticity, Dover Publications, 1996.

[2] F. Sheta, V.J. Harrand, D.E. Thompson, T.W. Strganac, Computation and experimental investigation of limit cycle oscillations of nonlinear aeroelastic systems, J. Aircr. 39 (1) (2002) 133-141.

[3] E.H. Dowell, J. Edwards, T.W. Strganac, Nonlinear aeroelasticity, J. Aircr. 40 (5) (2003) 857-874.

[4] C.M. Denegri, M.A. Cutchins, Evaluation of classical flutter analyses for the prediction of limit cycle oscillations, in: Proceedings of the 38th AIAA Structures, Structural Dynamics and Materials Conference, Orlando, FL, USA, 1997, AIAA Paper 97-1021.

[5] P. Chen, D. Sarhaddi, D. Liu, Limit-cycle oscillation studies of a fighter with external stores, in: Proceedings of the 39th AIAA Structures, Structural Dynamics and Materials Conference, Long Beach, CA, USA, 1998, AIAA Paper 98-1727.

[6] T. O'Neil, T.W. Strganac, Aeroelastic response of a rigid wing supported by nonlinear springs, J. Aircr. 35 (Jul-Aug 1998) 616-622.

[7] R. Vasconcellos, A. Abdelkefi, F. Marques, M. Hajj, Representation and analysis of control surface freeplay nonlinearity, J. Fluids Struct. 31 (6) (2012) 79-91.

[8] M. Conner, D. Tang, E. Dowell, L. Virgin, Nonlinear behavior of a typical airfoil section with control surface freeplay: a numerical and experimental study, J. Fluids Struct. 11 (1) (1997) 89-109. 
[9] S.T. Trickey, Global and local dynamics of an aeroelastic system with a control surface freeplay nonlinearity, PhD thesis, Department of Mechanical Engineering and Materials Science in the Graduate School of Duke University, 2000.

[10] A. Abdelkefi, R. Vasconcellos, F. Marques, M. Hajj, Bifurcation analysis of an aeroelastic system with concentrated nonlinearities, Nonlinear Dyn. 69 (1-2) (2012) 57-70.

[11] R.M. Vasconcellos, A. Abdelkefi, M.R. Hajj, D.P. Almeida, F.D. Marques, Airfoil control surface discontinuous nonlinearity experimental assessment and numerical model validation, J. Vib. Control (2014).

[12] A.H. Nayfeh, B. Balachandran, Applied Nonlinear Dynamics: Analytical Computacional, and Experimental Methods, Wiley Series in Nonlinear Sciences, John Wiley \& Sons, Inc, New York, 1995.
[13] T. Theodorsen, General theory of aerodynamic instability and the mechanism of flutter, tech. rep., NACA Report 496, 1935.

[14] D. Li, S. Guo, J. Xiang, Study of the conditions that cause chaotic motion in a two-dimensional airfoil with structural nonlinearities in subsonic flow, J. Fluids Struct. 33 (0) (2012) 109-126.

[15] M. Hajj, R. Miksad, E. Powers, Perspective: measurements and analyses of nonlinear wave interactions with higher-order spectral moments, J. Fluids Eng. 119 (1) (1997) 3-13.

[16] M. Hajj, R. Miksad, E. Powers, Fundamental-subharmonic interaction: effect of phase relation, J. Fluid Mech. 256 (1993) 403-426.

[17] M.R. Hajj, P.S. Beran, Higher-order spectral analysis of limit cycle oscillation of fighter aircraft, J. Aircr. 45 (Nov-Dec 2008) 1917-1923. 